\title{
Optical Measurement of Contact Forces using Frustrated Total Internal Reflection
}

\author{
James S. Sharp* and Stuart F. Poole \\ School of Physics and Astronomy, University of Nottingham, University Park, Nottingham, NG7 2RD, UK \\ Benjamin W. Kleiman \\ Adidas America, Adidas village, $5055 \mathrm{~N}$ Greeley Avenue, Portland, OR 97217, USA
}

(Dated: August 2, 2018)

\begin{abstract}
A simple device based on the principle of frustrated total internal reflection was used to image the regions of contact between rubber objects and a large area perspex waveguide. Measurements of the intensity of light scattered at the interface were found to depend upon the magnitude of the applied force, the mechanical properties of the contacting material and the roughness of the contacting objects. The intensity/force response was found to have the same functional dependence irrespective of the position on the waveguide surface, but to scale by an amount that was proportional to the local intensity of light incident on the perspex/object interface. Once this spatial variation in intensity had been calibrated, the waveguide could be used to perform optical measurements of the forces/pressures exerted on the surface of the waveguide and to generate spatial maps of the pressure at frame rates up to $200 \mathrm{~Hz}$. The resulting optical force platform was used to measure the time dependent evolution of the pressure distribution beneath a foot and a sports shoe during a foot strike event. A simple theory was developed to describe the light scattering phenomenon and to explain the relationship between the scattered light intensity and the applied force.
\end{abstract}

\section{INTRODUCTION}

Frustrated total internal reflection (FTIR) imaging is a simple optical technique which uses light to detect contact between an object and a surface. The design principles that underpin the technique are relatively simple. Light is totally internally reflected inside a slab of a transparent material such as glass or perspex (the waveguide) [1] which has a higher refractive index than the surrounding ambient material (see figure 1). Total internal reflection is achieved by ensuring that the angle of incidence of light hitting the interface between the slab material and the ambient is always greater than the critical angle, $\theta_{c}$. The condition which sets the critical angle is given by $\sin \theta_{c}=\frac{n_{a}}{n_{s}}$, where $n_{a}, n_{s}$ are the refractive indices of the ambient and slab materials respectively [2]. The boundary conditions experienced by the light being reflected inside the waveguides are such that evanescent waves are generated at the slab/ambient interface which propagate in the plane of the slab. The electric field amplitudes associated with these evanescent waves decay exponentially away from the interface and into the ambient material. This exponential decay of the field amplitudes occurs over distances on the order of the wavelength of the light that is used to excite the waveguide [2].

If an object is brought to within a wavelength or so of the slab, then it is possible for the evanescent waves to interact with the object in the regions of contact. There are two ways in which this can occur; either light will simply be transmitted into the material and escape the waveguide and/or it will be scatterred as a result of interactions with objects inside the contacting material.

\footnotetext{
* Corresponding author: james.sharp@nottingham.ac.uk
}

Transmission into the contacting material can occur when it has a higher refractive index than the ambient surroundings. Changing the refractive index at the boundary for a higher value increases the value of $\theta_{c}$ and some of the light that was previously incident at angles greater than the critical angle may now be allowed to escape the waveguide. In the case of scattering, the complex part of the refractive index adds an oscillatory component to the exponentially decaying fields associated with the evanescent wave and results in a propagating (scattered) waveform [3]. Both mechanisms result in light escaping from the waveguide and this can be detected by a camera or other detector placed either above or below the waveguide (depending on whether the light is transmitted or back scattered by the object). Under such conditions, the total internal reflection condition is said to have been frustrated by the presence of the object. This frustration occurs only in the regions where the object comes to within a wavelength or so of the surface, thus making the technique highly surface sensitive. As a result of this surface sensitivity, the technique has found a number of applications in the study of interfacial phenomena involving colloids and nanoparticles as part of what is more widely referred to as evanescent wave scattering [4].

The highly surface sensitive nature of FTIR imaging has also been exploited to image the contact between microscopic objects [5] and rough surfaces [6], to measure the gait of small animals [7] and insects [8]. It has also been used for the development of touch sensitive controllers [9], in the imaging of shoes for forensic applications [1] and fingerprint imaging for biometric identification $[10]$.

Betts and Duckworth [11, 12] were among the first to identify that FTIR can be used to measure contact forces. They observed an increase in brightness of the 
a)

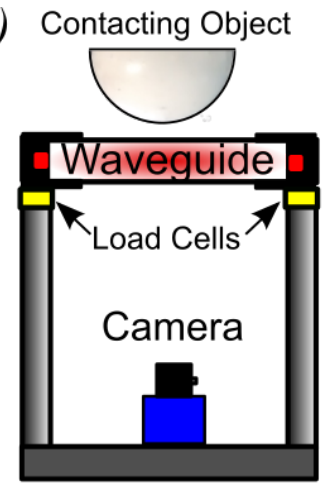

c)

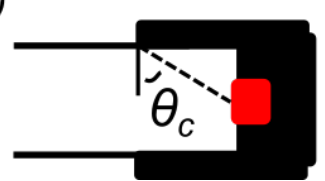

b) $5 \mathrm{~mm}$ dia puck

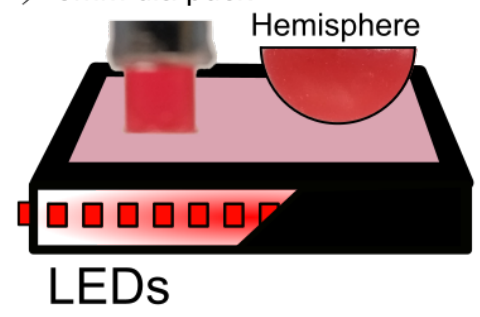

d)

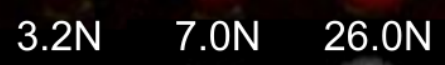

FIG. 1: Construction of the waveguide imaging element. a) Load cells were placed beneath a perspex waveguide and a camera used to image the interface between the waveguide and a contacting object. b) Ultrabright red LEDs were wrapped around the outside edge of the perspex. Cylindrical elastomer pucks and hemispheres were pressed into contact with the waveguide under varying axi-symmetric loads. c) The edges of the waveguide were masked with black tape to ensure that light incident on the perspex/air interface at an angle less than the critical angle, $\theta_{c}$ was not allowed to propagate inside the waveguide. d) A series of images showing the contact region observed by the camera in colour (top row) and in greyscale following background subtraction (bottom row). The corresponding values of the applied force are given next to each set of images. The scale bar is $2.5 \mathrm{~mm}$ in all of the images shown.

contact regions between human feet and a waveguide as the plantar pressure beneath the foot was increased. At the time, digital cameras were not readily available and processing the image data was difficult. However, recent advances in machine vision technology have meant that digital cameras and software have become relatively inexpensive. This has resulted in a renewal of interest in the use of the technique as a force sensor, with recent experiments to study the pressure distributions beneath car tyres [13] and geckos feet [14]. While these studies have demonstrated the feasibility of the technique, little has been done to explore the mechanisms that underpin the physics of FTIR imaging as a force sensor and how changing properties such as the colour, stiffness and surface roughness of the contacting objects influences the force/intensity response that is obtained.

It is most likely that the force sensitivity of the technique originates in variations in the degree of conformal contact between an object and the waveguide as an increasing load is applied. As the majority of surfaces are rough, total conformal contact is rarely achieved when two surfaces are pressed together. However, deformation of asperities on the surface of the objects leads to a gradual increase in the area of contact. In the context of FTIR imaging, this increase in area will lead to an increase in scattering intensity as the applied force is increased. Deformations in the material contained within the asperities will also lead to changes in the equilibrium separation of any scattering objects within them. This change in the local density of scatterers with varying strain is also likely to affect the intensity of light that is scattered.

The details of the contact mechanics process between two surfaces have been considered for a range of different contacting geometries and for surfaces with different roughness. Among the most simple of the theories is that of Hertzian contact mechanics [15]. This theory describes the evolution of the area of contact between two smooth surfaces having arbitrary radii of curvature. This is described in terms of the applied load as well as the Young's moduli and Poisson ratios of the contacting materials. While being useful for describing the macroscopic contact between surfaces, this simple theory fails to adequately describe the mechanics of contact on length scales that are comparable to that of the roughness of the surface. On these length scales, the contact mechanics of individual asperities have been demonstrated to be important in describing the true area of contact [6]. More sophisticated theories have therefore been developed to try to bridge the micro to macro scale gap in our understanding of contact mechanics processes. One of the more prominent theories which has proven useful in describing the contact between rough surfaces was presented by Greenwood and Williamson $[16,17]$. This theory considers the effects of roughness by modelling a surface as if it were covered in a series of asperities with a fixed radius of curvature. These spherical asperities have centres whose positions have a Gaussian distribution with respect to some average position (i.e. the surface itself). Hertzian theory is then used to describe the deformation of individual asperities and to predict the true area of contact when a known load is applied. A potential limitation of this theory is that it uses a single value for the mean radius of curvature to describe all the asperities on the surface. This means that detailed information about the distribution of radii of surface asperities needs to be obtained before a meaningful value of the average radius can be obtained.

In reality, surfaces have roughness that spans a broad range of different length scales and models that consider only a single length scale for roughness would appear to have limited applicability. A theory developed by Persson $[16,18]$ considers the entire spectrum of surface roughness and develops the details of the stress distribution beneath asperities at different magnifications. Persson's theory has been shown to give improvements in the predicted values of quantities such as the true area of contact and to be applicable over ranges of applied forces where the more simple theories break down.

The sensitivity of the FTIR contact imaging technique 
to changes in the area of contact clearly makes it useful for testing the predictions of the different contact mechanics theories. However, it also has potentially exciting applications for mapping the force/pressure distribution beneath objects with complex geometries such as hands and feet [11], tyres [13] and shoe out soles [1] if the details of the combined contact mechanics and light scattering processes can be better understood. In the case of sports shoes, observing areas of contact between the out sole and the ground can be instrumental in optimising footwear shape, tread pattern and stud placement [19].

Competing pressure plate technologies typically involve the use of mats of electrically addressable elements that are made of piezoelectric, resistive or capacitive devices [20]. These can be expensive and their spatial resolution is often limited by the size of the elements within them. In addition, they are often opaque and prevent optical access for visualisation of the contact regions. This is often desirable in contact mechanics experiments and for inspection purposes. In the case of FTIR imaging, the spatial resolution is set by the size of the pixels, the area of the sensor on the camera as well as the optics used to image the contact regions. All of these can be chosen to optimise the technique for a given application. Moreover, the imaging aspects of the technique allow for direct visualisation of the contact regions. However, one thing that has limited the use of the technique as a force/pressure measurement device has been a lack of detailed understanding of how the properties of the waveguide and the contacting material influence the intensity/force response.

In this study a simple light scattering theory is developed to try to predict the form of the intensity-force response when soft surfaces are pressed into contact with a solid perspex waveguide. Hertzian contact mechanics is used in combination with Rayleigh light scattering theory to predict the scattering response of individual asperities on a surface as they deform under an applied load. The theory is used to predict how the scattering intensity varies as a function of the applied load and the mechanical, physical and optical properties of the contacting surface. The predictions of this simple theory are compared to a series of contact mechanics experiments on elastomer samples with different surface roughness, Young's modulus and scatterer concentrations. The work is then extended to use the FTIR imaging technique to measure the pressure distribution beneath contacting objects with more complex geometries. These include spherical indenters, human feet and the out sole of a sports shoe. A high frame rate camera was used to map the force distributions beneath these objects at speeds of up to $200 \mathrm{~Hz}$ and to provide details of the evolving load pressure distribution under dynamic loading conditions.

\section{EXPERIMENTAL}

\section{A. Waveguide Construction}

The waveguide imaging element used in this work was prepared by wrapping ultrabright red LEDs (632 nm, RS Components Ltd \#153-3625) around the outside edge of a $200 \mathrm{~mm} \times 300 \mathrm{~mm} \times 15 \mathrm{~mm}$ piece of perspex as shown in figure 1. Prior to wrapping the LEDs, the edges of the perspex piece were roughened with abrasives to give uniform/diffuse scattering of the light. The LEDs were then tighly wrapped in such a way that they were separated by a distance of $15 \mathrm{~mm}$ around the periphery of the perspex. Once the LEDs had been attached, the perspex was wrapped in black electrical tape in such a way that it covered the outside edge and masked a small portion of the large faces (as shown in the figure 1). This was done to secure the LEDs to the edge of the perspex and to ensure that any light that was incident on the perspex/air interface at an angle smaller than the critical angle for total internal reflection was prevented from propagating inside the waveguide. The masked perspex element was then placed on top of four resistive load cells connected together in a bridge circuit and whose resistance was measured using a National Instruments USB6000 data acquisition (DAQ) card connected to a PC running LabView (National Instruments). A Basler ACE AC800-510uc USB 3.0 camera with a fixed focal length lens ( $\mathrm{f}=5 \mathrm{~mm}$, Computar) was then placed $170 \mathrm{~mm}$ below the waveguide and used to image its top surface through the perspex piece. The camera was also connected to the same PC as the DAQ card and images acquired using software written in LabView. The entire apparatus was mounted on a sturdy aluminium frame and was used for the majority of the experiments described below

A second larger waveguide having dimensions of 600 $\mathrm{mm} \times 600 \mathrm{~mm} \times 25 \mathrm{~mm}$ was also constructed in a similar way. Once again, $632 \mathrm{~nm}$ LEDs were wrapped around the periphery of a large piece of perspex, masked with black electrical tape and the whole assembly mounted on top of resistive load cells. An identical camera was used to image the top surface of the waveguide from beneath and a similar DAQ card was used to obtain force readings from the load cells. This second apparatus was used to image the contact between a foot and sports shoe outsole and the surface of the waveguide during dynamic loading experiments involving a foot strike event.

Under conditions of zero contact, the light generated by the LEDs remained confined inside the perspex waveguides as a result of total internal reflection at the perspex/air interface. However, when an object was placed in contact with the perspex, a region of scattered light was observed by the camera. Moreover, the intensity of the scattered light increased with the applied force. This occurred only in the regions where the object touched the waveguide (see figure 1). The presence of scatterers or dye molecules in the contacting object results in scattering of the exponentially decaying evanescent field. 
This changes the conditions for total internal reflection in the contact regions and results in a propagating scattered light wave which can be detected by a camera (see figure 1). The regions containing the scattered light were separated from any background by subtracting the blue and green channels from the red channel in the images that were acquired by the camera. This background subtraction procedure resulted in a series of 8-bit greyscale images whose pixel intensities were found to increase with the force applied to the surface of the waveguide (see figure 1d). While narrow band-pass optical filters could have been placed in front of the camera and used to image only the wavelength of interest (632 nm), this simple background subtraction technique proved to be more than adequate for removing the effects of background lighting.

\section{B. Sample Preparation}

Cylindrical pucks having a diameter of $5 \mathrm{~mm}$ and length $10 \mathrm{~mm}$ were prepared from Sylgard 184 Silicone (PDMS, Dow Corning) elastomer. The pucks were cast by mixing the Sylgard resin and crosslinker together with a commercially available red silicone dye (Polycraft red silicone pigment, MBFG) before pouring into $30 \mathrm{~mm}$ diameter cylindrical molds. The bottom face of the cylindrical mold was covered with abrasive (sand) paper having a range of different grit sizes in order to produce cured elastomer samples with different surface roughness values. All samples were cured at a temperature of $120^{\circ} \mathrm{C}$ for 1 hour in an oven at ambient pressure. The Young's moduli of the elastomer samples were varied by changing the resin:crosslinker ratio used to prepare the elastomer. The concentration of scatterers was varied by adding different quantities of dye to the mixtures. The $5 \mathrm{~mm}$ diameter pucks were cut from the larger cylinders using a cork borer. Measurements of surface roughness of the samples were performed using a zeta-20 optical profiler (Zeta Instruments) .

The Youngs moduli of the elastomer samples were measured using hemispheres of the same materials which had been cast into $30 \mathrm{~mm}$ diameter smooth hemispherical molds. The hemispheres were cured and pressed into contact with the waveguide surface using a metal plate attached to the back of the samples. Measurements of the area, $A$, of the circular contact patch that developed between the hemisphere and the waveguide were obtained as a function of the applied force, $F$, measured by the load cells (see figure 2). The Young's modulus of the elastomers, $E$, was extracted by plotting $F$ versus $A^{\frac{3}{2}}$ according the the predictions of Hertzian contact mechanics [15]

$$
F=\frac{4 E}{3 R\left(1-\nu^{2}\right)}\left(\frac{A}{\pi}\right)^{\frac{3}{2}}
$$

where $R$ is the radius of curvature and $\nu$ is the Pois- son ratio of the hemisphere (taken as $0.499[21,22]$ for Sylgard 184). Values of $E=0.475 \pm 0.002 M P a$, $0.811 \pm 0.05 M P a$ and $1.577 \pm 0.009 M P a$ were obtained for resin:cross linker ratios of 30:1, 20:1 and 10:1 respectively. These values were found to be in agreement with measurements obtained by other authors [21, 22].

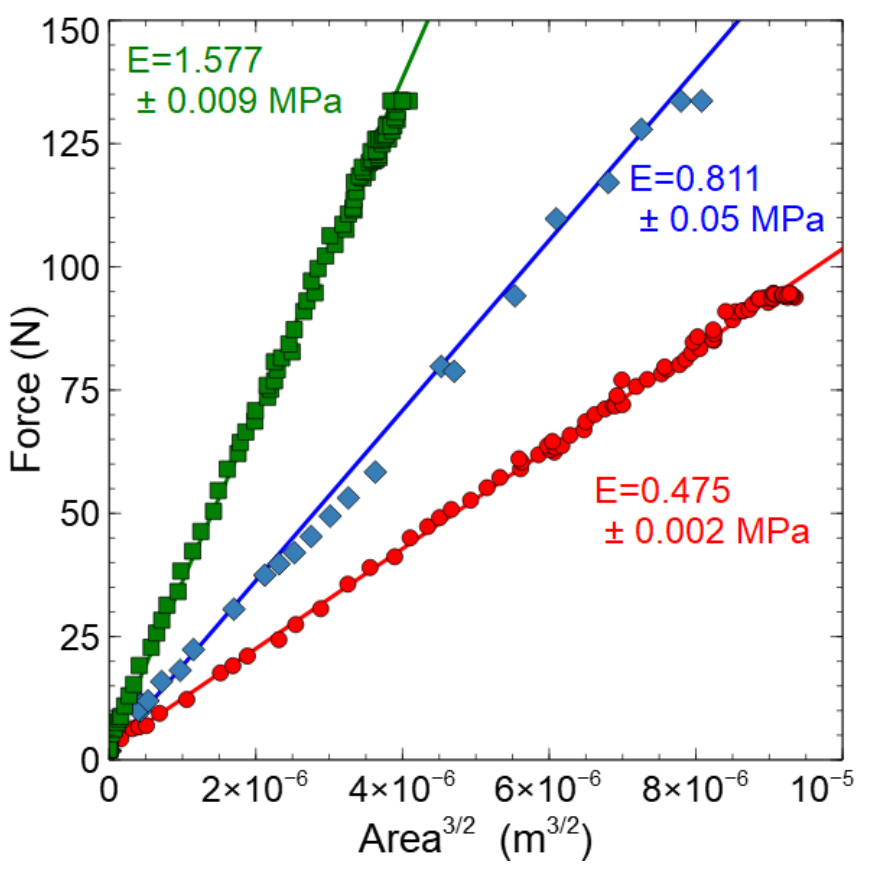

FIG. 2: Hertzian contact mechanics plots for dye doped elastomer hemispheres having resin:crosslinker ratios of 10:1 (green squares), 20:1 (blue diamonds) and 30:1 (red circles). The Young's modulus values of the dye doped elastomers were obtained from the slopes of the linear fits to these plots using equation 1 and are given as insets. The number density of dye molecules was $3 \times 10^{26} \mathrm{~m}^{-3}$ in each case.

Contact experiments were also performed using the $5 \mathrm{~mm}$ diameter elastomer pucks (see figure $1 \mathrm{~b}$ and $\mathrm{d}$ ). These samples were pressed into contact with the waveguide surface under varying loads. Care was taken to ensure that the applied forces were uniformly distributed across the surface of the pucks during loading. In each case, short movie sequences of the evolving contact were obtained and the average pixel intensity in the contact region was recorded by the camera. A series of images similar to those shown in figure 1d were obtained. Simultaneous measurements of the applied force were obtained using the signals from the load cells. Plots of the average intensity per pixel versus the average force per pixel similar to those in figure 3 were generated for pucks with different dye concentrations as well as different surface roughness and Young's modulus values. 


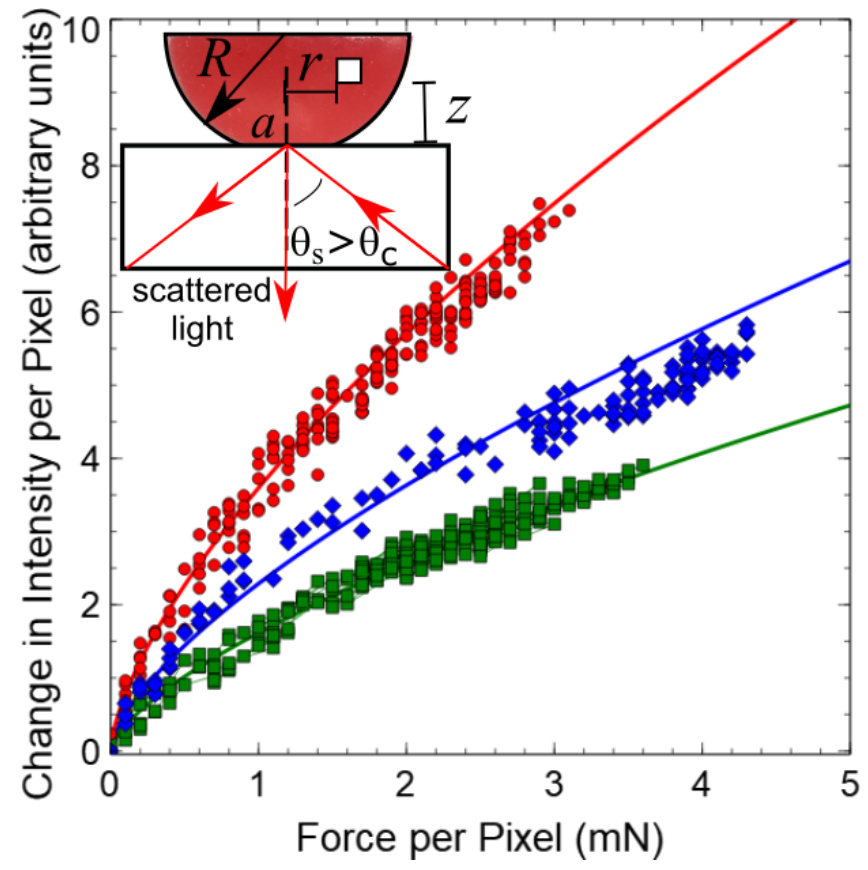

FIG. 3: Measured light intensity as a function of the applied load when $5 \mathrm{~mm}$ diameter cylindrical pucks were pressed into contact with the perspex waveguide surface. Data are shown for pucks with measured Young's moduli of $0.475 \pm 0.002 \mathrm{MPa}$ (red circles), $0.811 \pm 0.05 \mathrm{MPa}$ (blue diamonds) and $1.577 \pm 0.009 \mathrm{MPa}$ (green squares). The solid lines are simulations of the intensity/force response obtained from calculations using equation 10 with the same Young's modulus values. In each case the RMS roughness of the samples was $57.7 \pm 3.9 \mu \mathrm{m}$ and values of $R=50 \mu \mathrm{m}$ were used in the calculations. The number density of dye molecules was $3 \times 10^{26} \mathrm{~m}^{-3}$ in each case.

\section{Calibration of the Waveguides}

Some small amount of variation was observed in the waveguide scattering intensity due to natural variations in the intensity of light confined inside the waveguide and due to leakage caused by scratches and defects on the perspex surfaces. These combined factors caused the magnitude of the scattering intensity to vary with the position even though the shape of the intensity versus force curves was the same at different points on each waveguide surface. Natural variations in the waveguide intensity can be mitigated to some extent by changing its shape (e.g. using a circular waveguide rather than a rectangular one). However, these variations are stiil present and still need to be calibrated regardless of the waveguide geometry. The ease of manufacture associated with building a rectangular frame to support the waveguide used here far outweighed any small benefits that would have been gained by using a a waveguide with a different shape. Small scratches on the waveguide surface present potentially greater problems than effects due to the shape of the waveguide when it comes to introducing spatial variations in the scattering intensity. Scattering due to small surface scratches was often observed when viewing the waveguide at an oblique angle (i.e. away from normal incidence). However, the same scratches were not visible when viewed at normal incidence by the camera. This inability to detect the scratches when viewing at normal incidence was found to give the FTIR imaging technique a certain degree of resilience to small surface defects. However, the fact that these scratches scattered light away from the viewing direction of the camera caused small local variations in the scattered light intensity in the regions of the scratches which needed to be calibrated. In order to calibrate the spatial variations in the scattering intensity that were caused by both scratches and natural variations in the waveguide intensity, measurements of the scattering intensity per pixel versus the force exerted per pixel region similar to those shown in figure 3 were obtained at different positions on the waveguide surfaces. The scaling factor required to collapse all the intensity/force response recurves on to a sinlge master curve was recorded as a function of position and was used to correct the images to obtain a uniform intensity-force response when collecting data using the camera.

The area on the waveguide surfaces that was probed by each camera pixel was also determined. This was done by taking pictures of a ruler placed at different positions on the surfaces of the waveguides and measuring the number of pixels corresponding to a known length in the images. The area probed by each pixel was then calculated by squaring the length per pixel. Each pixel was found to correspond to an area of $0.033 \mathrm{~mm}^{2}$ on the surface of the smaller waveguide and $0.182 \mathrm{~mm}^{2}$ on the larger waveguide.

\section{RESULTS AND DISCUSSION}

The origin of the force sensitive light scattering phenomenon observed during frustrated total internal reflection can be interpreted in terms of changes in the conformity of contact between an object and the waveguide surface. Deformation of asperities on the surface of an arbitrarily rough object will lead to both an increase in the area of contact between an asperity and the waveguide as well as a change in the local density of scatterers close to the interface. The local contribution to the intensity, $d I_{s}$, of light scattered from a region within an asperity and measured by the camera can be obtained using the Rayleigh formula [23]

$$
d I_{s}=\frac{8 \pi^{4} I \alpha^{2}\left(1+\cos ^{2} \theta_{s}\right) N_{s}}{\lambda^{4} D^{2}}
$$

where $I$ is the intensity of light incident on the perspex/asperity interface, $\alpha$ is the volume polarisability of the scatterers in the object, $\theta_{s}$ is the scattering angle (see inset figure 3), $N_{s}$ is the number of scatterers upon which the light is incident, $\lambda$ is the wavelength of the 
incident light and $D$ is the distance between the camera and waveguide/asperity interface.

The intensity of light associated with the evanescent waves that are generated by total internal reflection at the interface between the asperity and the perspex waveguide can be written in the form

$$
I=I_{o} \exp \left(\frac{-4 \pi n_{o} \cos \theta_{r} z}{\lambda}\right)
$$

where $I_{o}$ is the value of the intensity at the waveguide/asperity interface, $n_{o}$ is the refractive index of the contacting asperity, $z$ is the distance inside the asperity as measured from the interface (see inset figure 3) and $\cos \theta_{r}$ is given by the relation

$$
\cos \theta_{r}=\left(\left(\frac{n_{w}}{n_{o}}\right)^{2} \sin ^{2} \theta_{i}-1\right)^{\frac{1}{2}}
$$

with $n_{w}$ being the refractive index of the waveguide and $\theta_{i}$ the angle of incidence $\left(=\theta_{s}\right.$ for the geometry chosen here, see inset figure 3 ).

The number of scattering objects in a small volume element at a distance $z$ from the interface, having a thickness, $d z$, can be written as $N_{s}=\phi A d z$ where $\phi$ is the local number density of scatterers and $A$ is the cross sectional area of the volume element. The number density, $\phi$, in the deformed asperity can be related to the number density in the undeformed asperity, $\phi_{o}$, and the local strain in the z direction, $\epsilon_{z}$, by the formula

$$
\phi=\frac{\phi_{o}}{1-(1-2 \nu) \epsilon_{z}}
$$

If each asperity on the surface of the contacting object is approximated as being a hemisphere of radius, $R$, the strain in the sphere can be obtained from the contact mechanics of spherical objects under axi-symmetric loading. If an axi-symmetric force, $F$, is applied to the spherical asperity this will result in a circular contact patch of radius, $a$ with a radially symmetric strain field inside the sphere, $\epsilon_{z}=\epsilon_{z}(z, r)$ of the form [15, 24],

$\epsilon_{z}=\frac{1}{E}\left((1-\nu) \sigma_{z}-2 \nu(1+\nu) \sigma_{o} \frac{z}{a}\left[\tan ^{-1}\left(\frac{a}{\sqrt{u}}\right)-\frac{a}{\sqrt{u}}\right]\right)$

where $E$ and $\nu$ are the Young's modulus and Poisson ratio of the asperity material respectively and

$$
\begin{aligned}
& u=\frac{1}{2}\left\{r^{2}+z^{2}-a^{2}+\sqrt{\left(r^{2}+z^{2}+a^{2}\right)^{2}+4 a^{2} z^{2}}\right\} \\
& \begin{array}{c}
\sigma_{z}=\sigma_{o}\left(\frac{z}{\sqrt{u}}\right)^{3} \frac{a^{2} u}{3 F^{2}+a^{2} z^{2}} \\
\sigma_{o}=\frac{3 a^{2}}{2 \pi a^{2}}
\end{array} \\
& a=\left(\frac{3 F R\left(1-\nu^{2}\right)}{4 E}\right)^{\frac{1}{3}}
\end{aligned}
$$

Combining equations 2 to 9 and using the fact that the cross sectional area of a small element at a radius, $r$, is $A=2 \pi r d r$, gives an expression for the total scattering intensity from a single asperity as

$I_{s}=\frac{16 I_{o} \pi^{5} \phi_{o} \alpha^{2}\left(1+\cos ^{2} \theta_{s}\right)}{\lambda^{4} D^{2}} \int_{0}^{\infty} \int_{0}^{a} \exp \left(\frac{-4 \pi n_{o} \cos \theta_{r} z}{\lambda}\right) \frac{r d r d z}{1-\frac{(1-2 \nu)}{E}\left((1-\nu) \sigma_{z}-2 \nu(1+\nu) \sigma_{o} \frac{z}{a}\left[\tan ^{-1}\left(\frac{a}{\sqrt{u}}\right)-\frac{a}{\sqrt{u}}\right]\right)}$

If each asperity occupies a region on the surface of area $\sim 4 R^{2}$ then the force exerted on a region of the surface that is imaged by a single pixel, $F_{p p}$, can be approximated using the expression

$$
F_{p p}=F \frac{A_{p}}{4 R^{2}}
$$

Similarly, the intensity measured per pixel, $I_{p p}$, can be written as,

$$
I_{p p}=I_{s} \frac{A_{p}}{4 R^{2}}
$$

where $A_{p}$ is the area of the surface of the waveguide that is probed by a given pixel on the camera.
The result obtained from calculation of the integrals in equation 10 can be compared directly to the results of the loading measurements obtained using the $5 \mathrm{~mm}$ puck samples. However, the intensity measured by the camera is converted to arbitrary units by the camera's on board A-D conveter. As such the integrals in equation 10 need to be multiplied by a constant value which captures this scaling. For all the data shown in figure 3, a single prefactor value of $3.3 \times 10^{16}$ was used to scale the results of the calculations for comparison with the data.

Equation 10 can be used to make predictions about the effects of changing parameters such as the Young's modulus, roughness (via information about the curvature of surface asperities) and the effects of changing dye concentration. The results of calculations based on equations 
10, 11 and 12 are shown in the plots in figure 3 for cylindrical pucks with different Young's modulus values. The calculations shown were performed using values of the Young's modulus that were the same as those measured in figure 2 and using a value of $R$ similar to the RMS roughness of the surfaces. The calculated $I_{p p}$ versus $F_{p p}$ curves were found to agree well with the measured data and to predict the correct dependence on the Young's modulus of the contacting material. The data and calculations both show that as the Young's modulus of the asperity material is decreased, the light scattering intensity increases. This is because low modulus materials deform more easily giving rise to a greater area of contact between each asperity and the waveguide (according to equation 1). Lower moduli also result in increased compressive strains being generated within the asperities when a given force is applied. These increased strains give rise to a reduction in the distance between scattering objects and hence an increase in the number density of scatterers (see equation 4 ). The combined effects are that a larger scattering intensity is obtained as the modulus is decreased (for a given applied force) and the surfaces are able to to attain conformal contact more easily.

The range of validity of equation 10 in describing the intensity/force response is set by limitations of the contact mechanics approach that is used to describe the deformations in the asperities. According to approximations made in Hertzian contact theory [15], the expression for the contact area is only valid when the contact radius is much smaller than the radius of curvature of the contacting object i.e. $a<<R$. In this limit, the equation which relates the force exerted on an asperity to the contact patch radius (equation 9) requires that equation 10 is only valid when $F<\frac{4 R^{2} E}{3\left(1-\nu^{2}\right)}$. Hence the approximations are only valid when the force exerted per pixel region obeys the condition that $F_{p p}<\frac{E A_{p p}}{3\left(1-\nu^{2}\right)}$. For the setup used here the upper bound on this force is $\sim 15 \mathrm{mN}$. The forces used in figure 3 are at least an order of magnitude smaller than this limit and fall well within this range.

The effects of changing the roughness of the elastomer pucks on the shape of the $I_{p p}$ versus $F_{p p}$ were also explored. Figure 4 shows plots of $I_{p p}$ versus $F_{p p}$ for elastomer pucks that were cured while in contact with different grades of abrasive paper. Elastomer surfaces with root mean square roughness values in the range $8.0 \pm 0.1 \mu \mathrm{m}$ to $57.7 \pm 3.9 \mu \mathrm{m}$ were prepared and studied. No systematic roughness dependence was observed in the $I_{p p}$ vs $F_{p p}$ curves shown in figure 4 . This result is perhaps counter intuitive at first but calculations using equation 10 also predict that the calculated $I_{p p}$ versus $F_{p p}$ curves are independent of the radius of curvature of the asperities. At high loading $\left(F_{p p}\right.$ greater than a few $\mathrm{mN}$ ) the calculated curves start to deviate from the measured curves due a breakdown in the validity of the approximations used in deriving the mechanical response of the asperities. However, the intensity/force response curves continue to behave in a similar way and have no

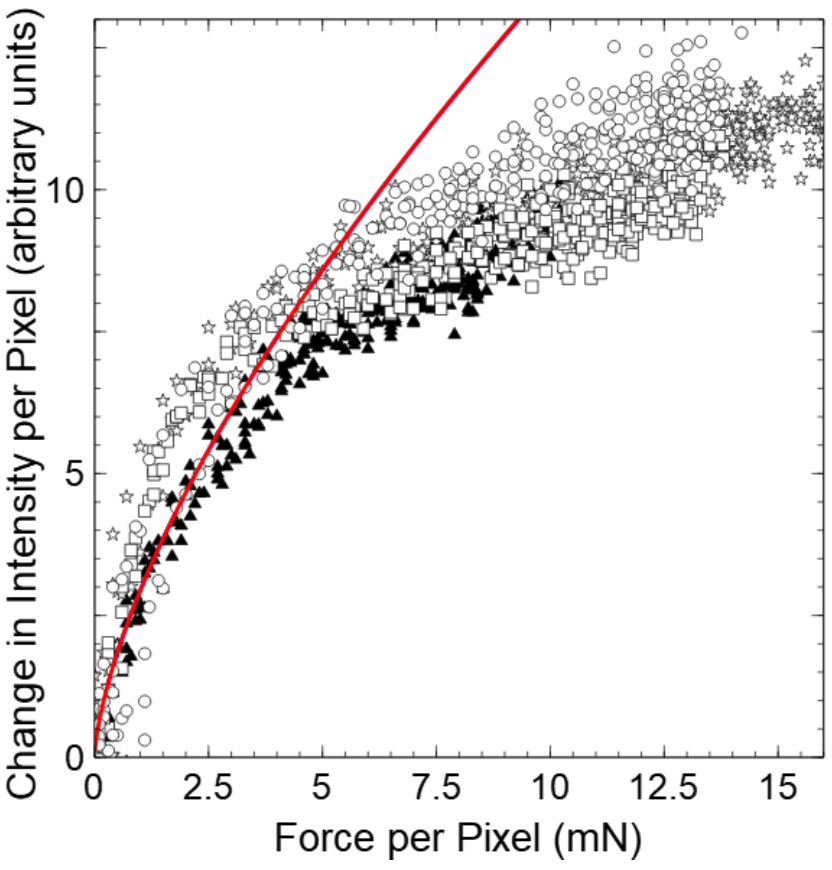

FIG. 4: Intensity/force response per pixel for elastomer pucks with different root-mean-square (RMS) roughness values. Data are shown for $5 \mathrm{~mm}$ diameter pucks with RMS roughness values of $8.1 \pm 0.2 \mu \mathrm{m}$ (white circle), $15.3 \pm 1.1 \mu m$ (white squares), $34.5 \pm 0.7 \mu m$ (white stars) and $57.8 \pm 3.9 \mu \mathrm{m}$ (black triangle). The red line shows the results of calculations based on equation 10 . The calculated intensity-force response per pixel is independent of the value used for the radius of curvature of the asperities for values. Calculations are shown for values in the range $0.01 \mu m<R<100 \mu m$. The Youngs modulus of the elastomer was $E=1.577 \pm 0.009 \mathrm{MPa}$ and the number density of dye molecules was $3 \times 10^{26} \mathrm{~m}^{-3}$ for each of the samples.

systematic dependence on RMS roughness.

The assumptions made here in relating a surface covered in asperities with a fixed radius of curvature to the RMS roughness are rather simplistic. Real surfaces have roughness on a broad range of length scales and capturing the contact mechanics of such surfaces using more sophisticated and elegant models has been the result of work by previous authors [16-18]. However, while the RMS roughness and radius of curvature of the surface asperities are not exactly equivalent, they are related increasing the radius of curvature of surface features will give larger RMS roughness values. Hence the prediction that the intensity/force response per pixel is independent of the radius of curvature of the surface features appears to be consistent with the lack of systematic roughness dependence observed in figure 4 .

A more simplified version of the scattering theory which approximates the strains in the asperity using near surface displacements can be used to obtain an approximate analytical solution for the intensity/force response 
measured by an individual pixel on the camera (see appendix A). This simplification of the theory gives further insights into the lack of sensitivity of the FTIR imaging technique to variations in roughness. It predicts that the scattering intensity from a single asperity (equation A3) depends upon the square of the radius of curvature of the surface features. However, when the average intensity per pixel is calculated using equation 11 , the dependence upon $R$ disappears (see equation $\mathrm{A} 4$ ). The physical interpretation of this result is based upon differences in areal number density of asperities with different radii of curvature. Asperities with small radii of curvature will have larger areal number density than larger ones. As a result, any reduction in scattering intensity that arises due to a smaller asperity size will be compensated by the higher number density when averaging over large areas of the surface. Hence, differences in the areal density of asperities with different radii of curvature result in a scattering intensity which does not depend upon the local roughness/radius of curvature when the contributions from many asperities are averaged in a single pixel. In the limit of small forces, the simplified version of the theory also predicts that the scattering intensity per pixel has a simple power law dependence on the applied load and predicts that the slope of the $I_{p p}$ versus $F_{p p}$ varies inversely with the Young's modulus of the contacting material (see equation A5). Again this is consistent with the results presented in figure 3 .

Both equations 10 and A4 also make predictions about the influence of changing the dye concentration on the shape of the $I_{p p}$ versus $F_{p p}$ plots. According to both these equations, the dominant contribution to the scattering intensity should arise due to the factor of $\phi_{o}$ in the numerator. Figure 5 shows three different $I_{p p}$ versus $F_{p p}$ plots that were generated using elastomer pucks with dye concentrations that vary by a factor of 100 . The inset to this figure shows a plot of the prefactor that was used to scale the calculations performed using equation 10 in order to obtain the best fits to the data. As the inset shows, the dependence is such that the prefactor/multiplier that was used increases with dye concentration, but it is not linear in $\phi_{o}$. The lack of linearity in $\phi_{o}$ that is observed in the measurements could represent a limitation of the theory introduced above. However, there may also be other reasons why the scattered light intensity may not scale linearly with the dye concentration. One possible factor that has not been accounted for could lie in the mechanism responsible for the exponentially decaying light intensity inside the contact regions. For example, increasing the dye concentration could be expected to influence the penetration depth via changes in the refractive index of the elastomer. Any such concentration dependence would influence the prefactor in equation A4 via $n_{o}$ in the denominator. However, measurements of the refractive index of the elastomer performed using an Abbe refractometer showed that the real part of the refractive index of the elastomers did not vary significantly with the concentration of dye.
It should be mentioned that evanescent waves are not the only way that an exponentially decaying intensity profile, similar to that in equation 3 , can be obtained. A propagating wave solution is also capable of generating an exponentially decaying waveform when scattering or absorption occurs in the material. Both of these mechanisms will attenuate an incoming light wave and will give rise to an exponentially decaying intensity whose length scale is set by the attenuation coefficient/complex part of the refractive index [3]. Such a mechanism would not influence the form of the equations presented here but it would change the magnitude of the decay length in equation 3. The net result of this other mechanism would be to replace the real part of the refractive index $n_{o}$ with the imaginary part of the refractive index in the denominator of equation A4. The complex part of the refractive index is expected to depend upon the concentration of dye in the elastomer (via the Lambert-Beer law) and hence this would modify the concentration dependence of the scattering intensity predicted by equations 10 and A4.

It is feasible that some of the light incident on the perspex/asperity interfaces used here could have been transmitted into the asperities and scattered. The change in boundary conditions caused by bringing the elastomer into contact with the waveguide results in a change in the critical angle from that of the perspex/air interface $\left(\theta_{c} \sim 42^{\circ}\right)$ to a new value of $\sim 71^{\circ}$ based upon the PDMS and perspex having refractive indices of 1.41 and 1.495 [25] respectively. The masking of the waveguide used in these experiments was such that that all angles of incidence greater than $\sim 69^{\circ}$ were allowed to propagate inside the waveguide under conditions of zero contact. Some small proportion of the light with angles of incidence between $69^{\circ}$ and $71^{\circ}$ could therefore have been refracted into the contacting objects and scattered via this mechanism.

An alternative cause of the weaker than expected concentration dependence in figure 5 could have been generated by excess scattering around the periphery of the pucks. As shown in the insets in figure 5 the edges of pucks with the lowest dye concentration demonstrated larger amounts of scattering than was observed in the central regions. When averaged over the entire contact region, this has the effect of increasing the average scattering intensity per pixel. The reasons for the nonuniform distribution of the scattering intensity observed in these samples are not entirely clear. However, such an effect could arise if the samples were slightly concaved, or if they experienced a degree of surface buckling when pressed into contact with the waveguide surface.

What is clear from the experiments and calculations performed above is that $I_{p p}$ versus $F_{p p}$ curves have a self similar shape that scales with Young's modulus, the concentration of scattering objects and the optical properties (refractive index and volume polarisability) of the contacting material. The predicted scaling of the intensity/force response on these parameters is such that the force sensing technique should also work with other mate- 


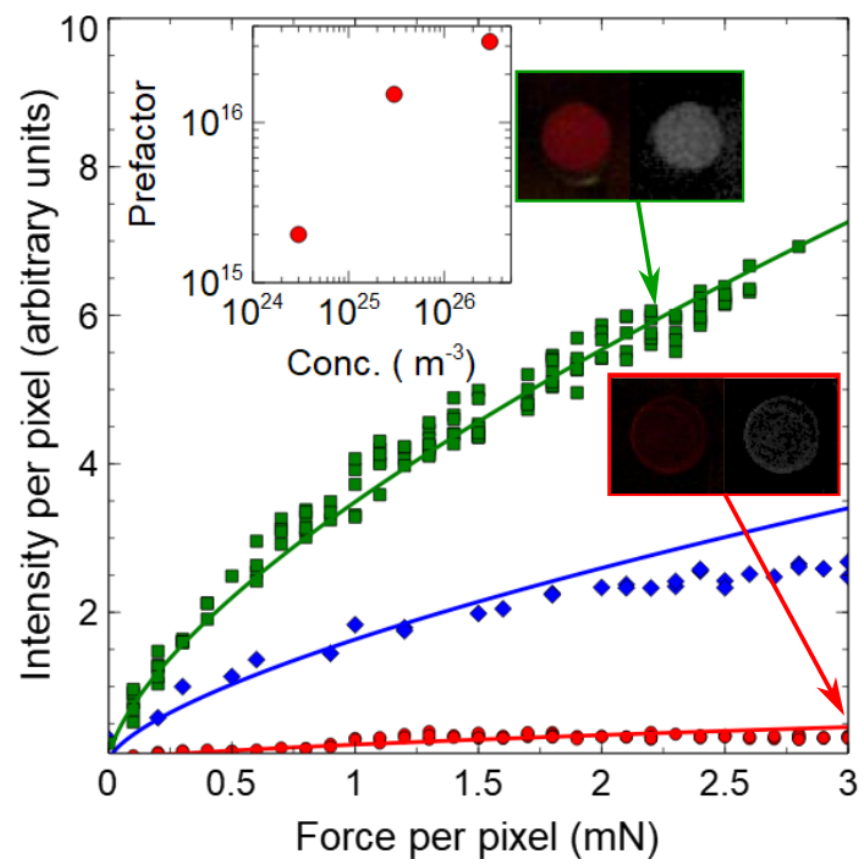

FIG. 5: Intensity/force response per pixel for elastomer pucks with different dye concentrations. Data are shown for $5 \mathrm{~mm}$ diameter pucks having number densities of dye molecules of $3 \times 10^{26} \mathrm{~m}^{-3}$ (green squares), $3 \times 10^{25} \mathrm{~m}^{-3}$ (blue diamonds) and $3 \times 10^{24} \mathrm{~m}^{-3}$ (red circles). The Youngs modulus of the elastomer was $E=0.475 \pm 0.002 \mathrm{MPa}$ and the RMS roughness was $39.1 \pm 8.9 \mu \mathrm{m}$ for each of the samples studied. The solid lines represent the results of calculations performed using equation 10 with the same values of $E$ and $R$. The inset shows a plot of the prefactor that was used to scale the calculations to fit the data as a function of the number density/concentration of the dye.

rials. In fact, the above analysis implies that the amount of light scattered by a contacting object should scale in a similar way regardless of the type and colour of the material being brought into contact, providing that it deforms more readily than the waveguide material.

The details of the sensitivity/prefactor in the limit of small forces will be determined by the Youngs modulus, optical properties and local intensity of the light incident on the waveguide according to equations 10 and A4. This implies that if the spatial response of the intensity of the waveguide can be calibrated across its surface, measurements of the intensity/force response can be used to calibrate the force sensing properties of the device for each new material. As such, each pixel on the camera can be used as an individual force sensor. Once each pixel has been calibrated the scattering from the waveguide obtained during contact can be used to map the force/pressure distributions beneath more complex objects than the flat elastomer pucks described above. Moreover, if a high frame rate camera was used, these images could be acquired quickly enough to perform dy-
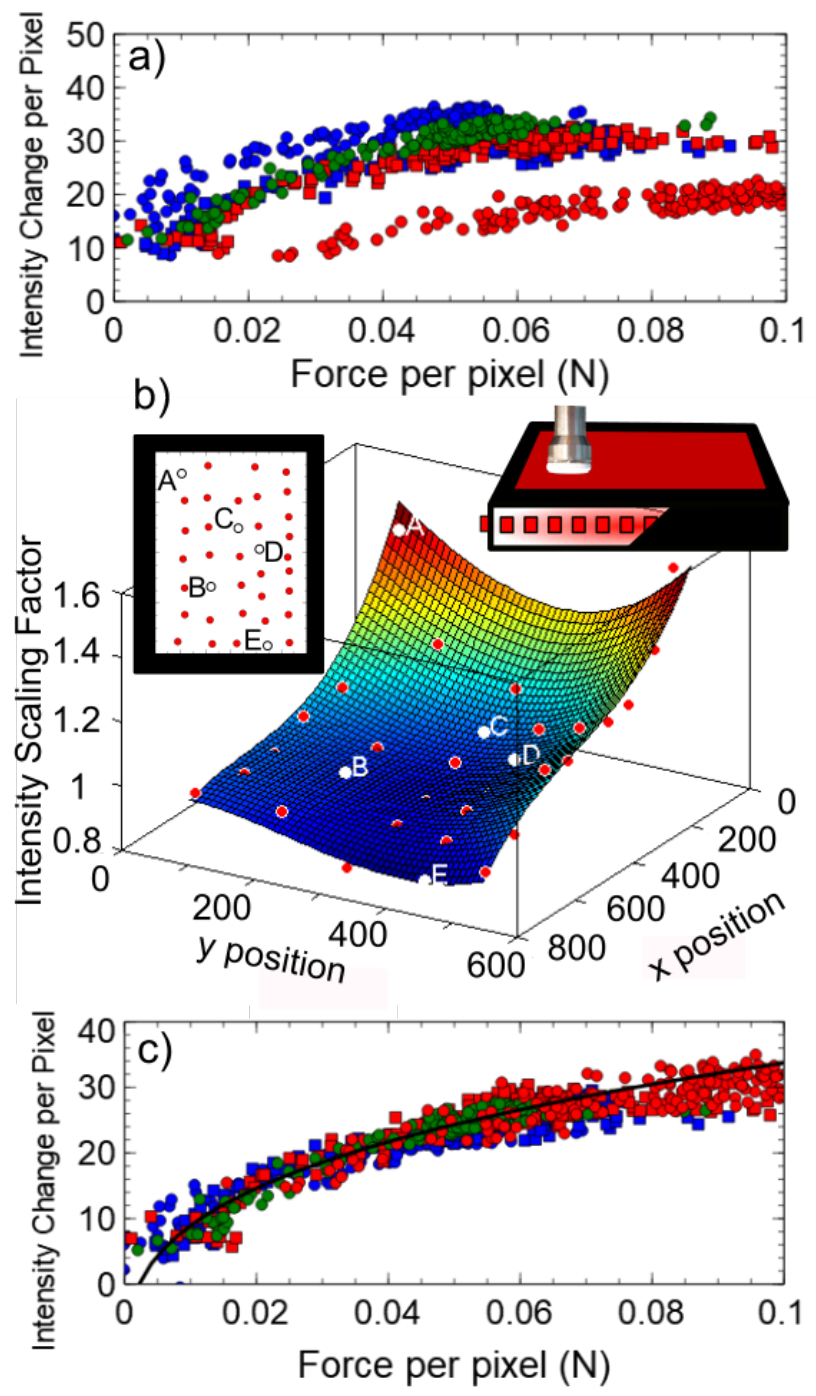

FIG. 6: Calibration of variations in intensity/force response at different positions on the waveguide surface. Panel a) shows measurements of the intensity per pixel vs. the force per pixel at positions A (red circles), B (green circles), C (red squares), D (blue squares) and E (blue circles) when the small flat punch was pressed into contact with the waveguide surface (see insets panel b). The main plot in panel b) shows how the scaling factor required to collapse the intensity-force curves onto a single master curve (panel c) varies as a function of position on the waveguide. The red data points in panel b) represent scaling factors for intensity-force curves measured at each position shown. The surface plot represents a 3rd order polynomial surface fit to the scaling factor data. The fit to the scaling factors was used to calibrate out spatial variations in the waveguide response as shown in panel c). The collapsed data sets were then fitted to the functional form given by equation 14 (solid line). 
namic measurements of the evolving force/pressure distribution beneath a soft object.

To test this hypothesis and to see if the approach described above works with other materials and waveguides, a separate, larger perspex waveguide having dimensions of $600 \mathrm{~mm}$ x $600 \mathrm{~mm}$ x $25 \mathrm{~mm}$ was used. Red (632 nm) LEDs were wrapped around its outer edges and were masked in a similar way to that shown in figure 1. A number of samples including a $20 \mathrm{~mm}$ diameter, white cylindrical polyurethane (PU) foam punch, a series of coloured PU hemispheres and a white rubber hemisphere were pressed into contact with the top surface of the waveguide using an axi-symmetric loading geometry. This ensured that the pressure distributions beneath the samples were radially symmetric during loading. The contact regions between the objects and the waveguide were then imaged using the camera (see figure 1) and the total force simultaneously measured using load cells. Background subtraction in the images was again performed by subtracting the blue and green colour channels from the red colour channel to produce greyscale images containing only the regions of contact.

In order to calibrate the intensity/force response of the new waveguide, the small cylindrical (PU) punch was pressed into contact with the waveguide at different positions on its surface and the grey scale intensity measured as a function of the applied load at multiple $x$ and $y$ positions. A similar calibration procedure was also performed on the smaller waveguide. In each case, the average intensity per pixel, $I_{p p}(x, y)$, in the contact region was plotted against the average force exerted per pixel, $F_{p p}(x, y)$. The intensity/force response was found to have a similar functional form (see figure 6a) to that observed with the dye doped elastomer pucks on the smaller waveguide. However, the slope of the curves was found to be different to that obtained from the red elastomer pucks and to vary by a simple numerical scaling factor that depended upon the position on the waveguide surface. The magnitude of the intensity scaling factor, $k(x, y)$, required to collapse all the intensity/force response curves taken at different positions (figure 6a) on to a single master curve (figure 6c) was determined at each point on the waveguide surface. The spatial variation of this scaling factor was fitted to a third order polynomial surface (see figure $6 \mathrm{~b}$ ) and used to calibrate the intensity variations across the waveguide surface. This calibration was performed by multiplying the grey scale intensities in each pixel in the image such that the new intensity, $I_{m}(x, y)$ was given by

$$
I_{m}(x, y)=k(x, y) I_{p p}(x, y)
$$

In this way, the positional dependence of the intensity scaling factor, $k(x, y)$, was used to correct the greyscale images so that the intensity/force response was the same at all points in the images. Having calibrated the spatial intensity response of the waveguide, the imaging device could be used to extract forces using a fit to the master curve shown in figure $6 \mathrm{c}$ of the form

$$
F_{p p}(x, y)=\beta \frac{I_{m}(x, y)^{a}}{b}
$$

where $a$ and $b$ are constants. The value of the power that best fit the data over the entire force range was found to be $a \approx 3$. This corresponds to $\frac{1}{3}$ power law dependence for the intensity/force relationship shown in the fit in figure $6 \mathrm{c}$. This is clearly different to the $\frac{2}{3}$ power law dependence that is predicted by the simplified form of the light scattering theory. However, it is worth recalling that equation A5 is only valid in the limit of small contact forces. It is therefore not surprising that a different power law can be used to parameterise the intensity/force response over the entire range of forces studied here.

The parameter, $\beta$, in equation 14 contains information about the dependence of the scattering intensity upon the Young's modulus of the contacting object as well as details about the local density of scatterers and information about the optical response of the material at the wavelength used to excite the waveguide $(632 \mathrm{~nm})$. In the case of the polyurethane punch, the value of $\beta$ was arbitrarily set to a value of one and all other $\beta$ values for the different materials were scaled relative to this value.

The calibrated waveguide was used to measure the contact beneath a number of polyurethane foam rubber and white rubber hemispheres with different colours, sizes and mechanical properties (see table I and inset figure 7). These samples were chosen because the pressure distribution beneath a contacting sphere has a nonuniform, but well-defined radial dependence. The hemispherical samples were therefore used to determine if the optical technique can be used to reliably measure the forces/pressures beneath objects with more complicated geometries than the simple flat punch used in the calibration measurements. In each case, the hemispheres were pressed into contact using an axi-symmetric loading technique and the force measured by the load cells was recorded at a rate of 200 samples per second. Images of the contact regions between the spheres and the waveguide were also measured at the same time points as the force by collecting movies of the evolving contact regions at 200 frames per second. The collection of the movies was triggered at the same time as the load cells to ensure synchronisation of the two measurements. The intensities at each position in the grey scale images were then used to extract values of the optically derived force at each position, via equation 14 .

The total optically derived force, $F_{\text {opt }}$, was then calculated by summing over all the pixels in the contact region, such that,

$$
F_{o p t}=\Sigma F_{p p}(x, y)
$$

The top panel in figure 7 shows a plot of the optically derived force as a function of the force measured using the load cells for a number of the materials described in table 
TABLE I: Properties of the materials used in the FTIR imaging contact mechanics studies (see text)

\begin{tabular}{|c|c|c|c|c|}
\hline Ball Type & Diameter $(\mathrm{mm})$ & Force Scaling Factor $(\beta)$ & $\mathrm{E}(\mathrm{kPa})$ & Measured Density $\left(\mathrm{kgm}^{-3}\right)$ \\
\hline Yellow PU Foam & $90 \pm 1$ & 0.1333 & $48 \pm 5$ & $70 \pm 2$ \\
\hline Orange PU Foam & $90 \pm 1$ & 0.1577 & $98 \pm 8$ & $73 \pm 2$ \\
\hline Pink PU Foam & $70 \pm 1$ & 0.1667 & $102 \pm 6$ & $101 \pm 3$ \\
\hline Green PU Foam & $70 \pm 1$ & 2.8 & $197 \pm 5$ & $105 \pm 3$ \\
\hline White Rubber & $38 \pm 1$ & 0.05 & $156 \pm 2$ & $1080 \pm 20$ \\
\hline
\end{tabular}

I. The value of $\beta$ was initially arbitrarily set to one for all the materials studied to determine how changing the optical properties influenced the relationship between the optically derived force and the force measured using the load cells. For all of the materials studied, the optically derived force was found to be linear in the load cell force and the gradient of the plots was found to be different for each material. The inset in the top panel of figure 7 shows a plot of the rescaled optical force vs load cell force. In each case, the curves were scaled by a parameter, $\beta$, in such a way that the value of the optical force was equal to that of the load cell force (see table I). The bottom panel in figure 7 shows plots of the reflectance spectra of the different ball materials that were measured using an Ocean Optics Red Tide USB spectrometer. This plot also shows the spectral response of the LEDs that were used to excite the waveguides and identifies the spectral region that is important for understanding the light scattering response in these experiments. The inset also shows a plot of the $\beta$ parameter that was used to rescale the optical force data as a function of the reflectance measured from each surface at $632 \mathrm{~nm}$. What is apparent from these data is that materials which scattered strongly in the region of the visible spectrum close to the excitation wavelength of $632 \mathrm{~nm}$ were found to have smaller values of $\beta$, while materials that did not scatter strongly were found to require significantly larger scaling values. This confirms that there is a strong correlation between the $\beta$ parameter and the optical properties of the ball in addition to any other contributions from Young's modulus and scatterer concentration. The data obtained from the samples that demonstrated weak scattering in the LED spectral region also contain significantly more noise. This suggests that choice of appropriate waveguide excitation wavelengths is therefore be important for obtaining the best scattering response from materials of a given colour.

The fact that all the curves in figure 7 vary linearly with the load cell force confirms two things about the FTIR imaging technique. Firstly, the intensity/force response (per pixel) is independent of the shape of the contacting object being pressed into the waveguide. Secondly, the shape of the $I_{p p}$ versus $F_{p p}$ curves is the same for each different material, but with a sensitivity that is detemined by the physical properties of the contacting material. These combined effects suggest that other contacting objects with different and more complex geometries can be studied using the FTIR imaging technique if a simple calibration is performed to determine the value of the $\beta$ parameter for each new material.

To further test these ideas, the contact imaging technique described above was used to extract information about the spatial distribution of forces/pressure beneath the contacting hemispheres. The pictures shown in the insets in figure 8 show the variations in intensity in the grey scale images that were collected from the contact regions between a $90 \mathrm{~mm}$ diameter orange polyurethane foam hemisphere and the waveguide surface. These were collected at different applied loads during an axisymmetric loading experiment. As these images show, the intensity is higher in the centre of the contact regions and decays radially outwards.

Equations 13 and 14 were used to convert the intensity values in these images and to extract the force exerted on each pixel. These values were then divided by the area of a pixel and radially averaged to produce the plots shown in the main panel of figure 8 .

As these plots show, the radially averaged pressure decays away from the centre of the contact regions. The solid lines in the main panel are fits to the radial pressure distribution, $P(r)$, using the functional form predicted by the Hertzian contact theory for a sphere being pressed into a flat hard surface [15],

$$
P(r)=\sigma_{o}\left(1-\left(\frac{r}{a}\right)^{2}\right)^{\frac{1}{2}}
$$

where $\sigma_{o}$ is the pressure at the centre and $a$ is radius of the contact region. The agreement between the data in figure 8 and the fits to equation 16 is good and indicates that the FTIR technique is indeed capable of extracting information about spatial variations in the contact forces/pressures beneath objects with non-planar geometries.

Measurements of the total optically derived force obtained from the contacting hemispheres were also used to try to extract information about the mechanical properties of the different hemisphere materials. Figure 9 shows plots of the optically derived force (after correction by the material dependent $\beta$ factor) as a function of the cube of the contact radius, similar to those shown in figure 2. This was repeated for all the hemispheres described in table I. According to Hertzian contact theory (equation 9), such plots should be linear with a slope that is proportional to the Youngs modulus of the sphere and inversely proportional to its radius. The slopes of the 


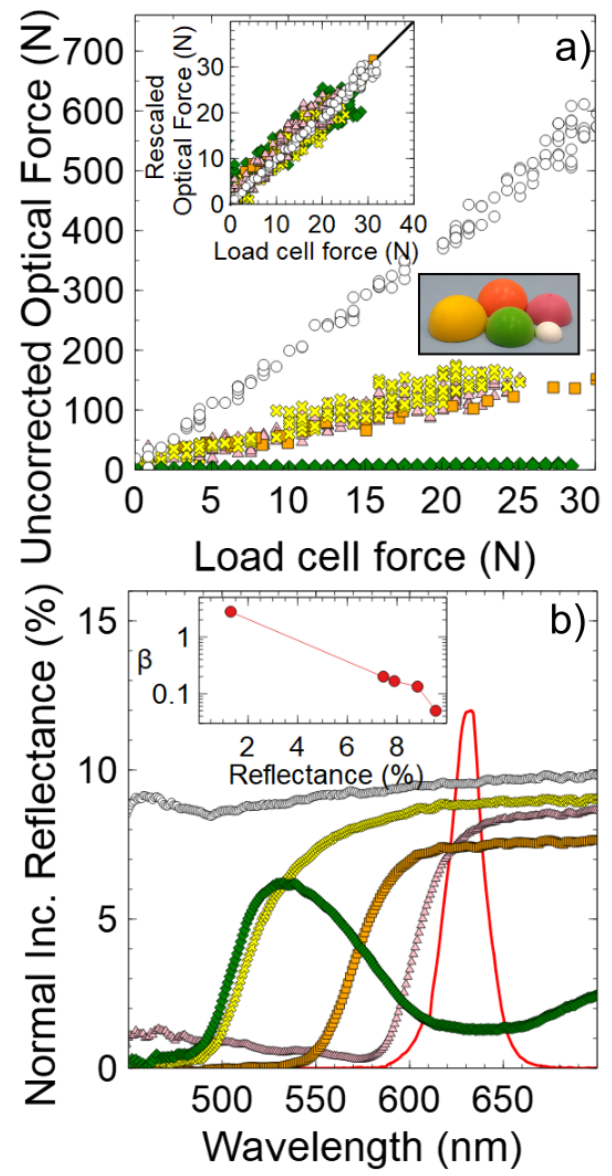

FIG. 7: Variation of the optically derived force with the force measured by the load cells. Panel a) shows data taken from a soft white rubber hemisphere (circles), as well as green (diamonds), yellow (crosses), pink (triangles) and orange (square) foam rubber hemispheres. The colour of the data points correspond to the colour of the hemisphere used in each experiment (see table I). The inset in panel a) shows the same data after it has been rescaled by a material dependent parameter, $\beta$ (see text). The second inset shows a photo of the balls used. Panel b) shows normal incidence reflectance spectra for each of the materials. The data sets are coloured according to the scheme used in panel a). The red line shows the (scaled) spectral response of the LEDs that were used to excite the waveguide. The inset shows a plot of how the $\beta$ parameter varies with the reflectance measured at the peak emission wavelength of the LEDs.

curves shown in figure 7 were used in combination with the data given in Table I and fits to equation 1 in order to extract the Youngs modulus, $E$, of the ball materials. The resulting values of $E$ are also summarised in table I. When calculating the Youngs modulus of each material, a value of 0.5 was used for the Poisson ratio. This value is consistent with that of most rubbers [25] and was deemed to be a suitable choice for the samples studied here. As expected, the Youngs modulus values

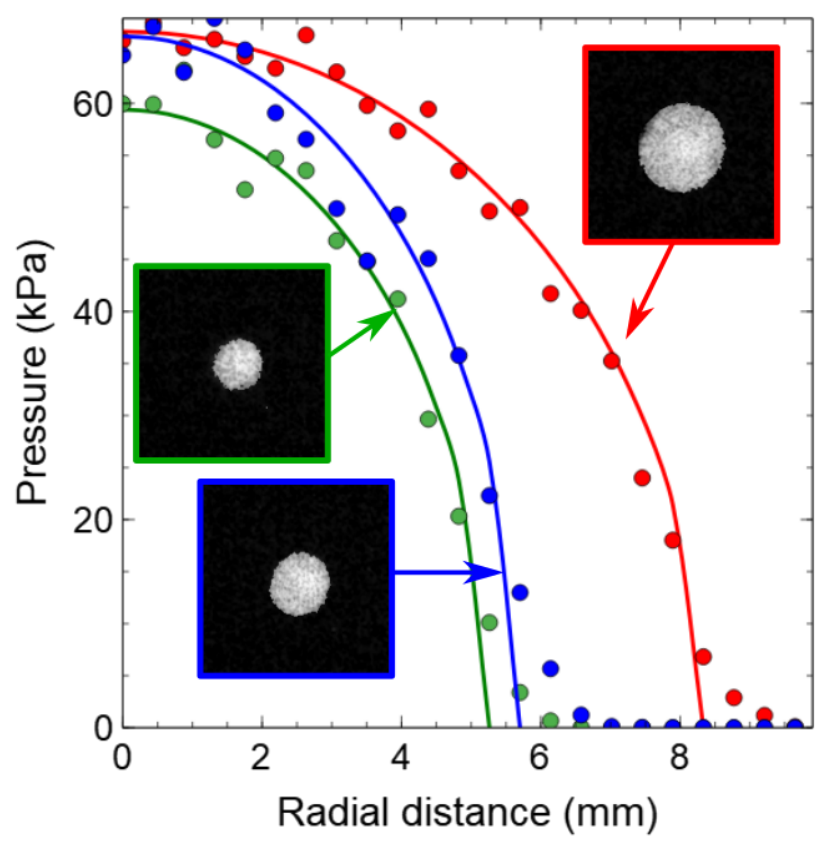

FIG. 8: Radial pressure distribution beneath a hemisphere. The main panel shows plots of the radially averaged pressure extracted from FTIR contact images of the orange hemisphere (shown as insets). The data points show the pressure values that were extracted from the images when the total applied force was $3.4 \pm 0.1 N$ (Green), $5.1 \pm 0.1 N$ (Blue) and $10.7 \pm 0.1 N$ (Red). The borders on the images are colour coded to match the datasets. The solid lines are fits to the form of the pressure distribution that is predicted by the Hertzian contact theory (equation 16).

obtained from these experiments were found to increase with increasing density of the foam and to be consistent with those measured for similar materials [26].

Having developed confidence in the ability of the FTIR technique to measure the force/pressure distribution beneath more complex objects, the larger waveguide was used to image the interactions between a bare foot and its surface (figure 10a) as well as a shoe outsole and its surface (figure 10b) during foot strike events. Movies of the contact regions were obtained at 100 frames per second as one of the authors stepped up on to the surface of the waveguide. Examples of the movies that were collected are included in Video 1 and Video 2. The colour movies/images that were acquired using the camera were background corrected and the grey scale intensity used to calculate the force exerted on each pixel using equation 14. Movies showing maps of the force distribution exerted beneath the foot and shoe were then generated using the calculated force values for each pixel (see Video1 and Video 2 for examples of movies and figure 10 for individual movie frames). The total force was then determined by summing the optically derived forces over all the pixels (equation 15). This was plotted on the 


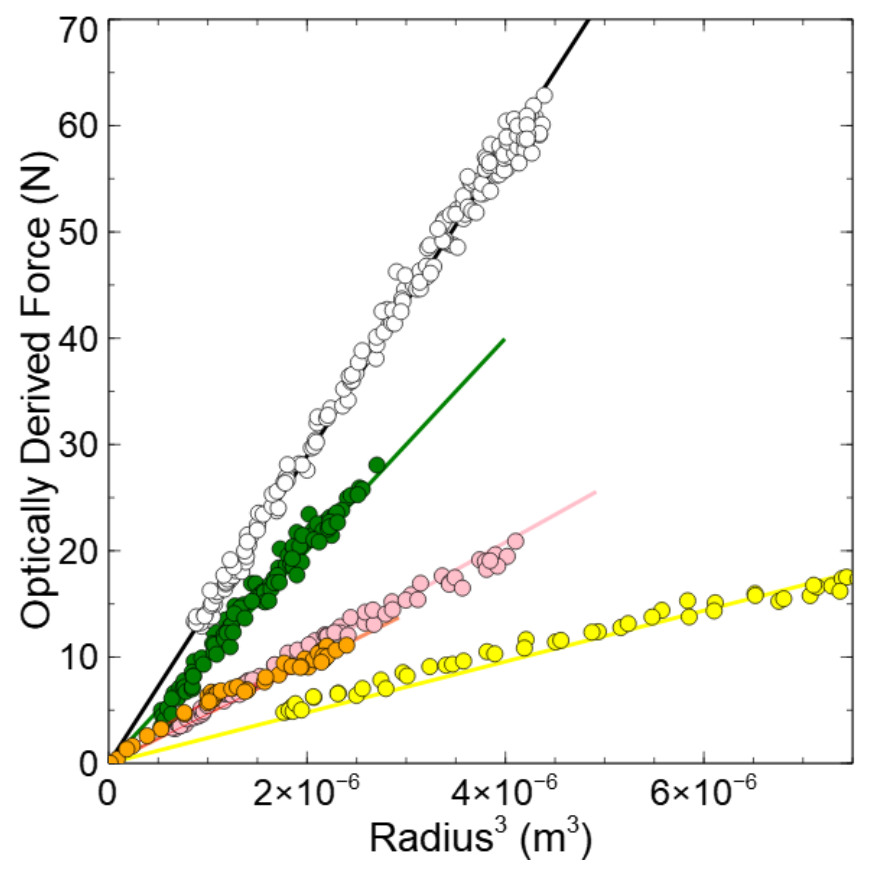

FIG. 9: Hertzian contact mechanics. Data are shown for the total optically derived force, $F_{O P T}$, plotted as a function of the cube of the radius of the contact region. Green, yellow, pink and orange foam rubber hemispheres and a white rubber hemisphere were used in these experiments. The data points are coloured to match the colour of the hemisphere used. The solid lines are fits to the first term of the Hertzian contact equation (equation 1). A summary of the results of these fits is given in Table I.

same axes as the force measured using the load cells as a function of time. Figure 10 shows that the measurements of the total optically derived force and the load cell force are in good agreement. The individual movie frames also provide detailed information about how the load was distributed beneath the foot and shoe at each time point.

This final set of experiments demonstrates the versatility of the FTIR based force sensing approach as a means of acquiring dynamic information about the changing force/pressure distributions between a contacting object and a waveguide surface. The design of the waveguide imaging apparatus is extremely simple - requiring only a piece of perspex, some LEDs and an inexpensive $(\approx 400$ GBP) camera - and can be incorporated easily into a number of different experimental geometries and environments. There are numerous potential applications for the FTIR force sensing technique including the development of touch screen sensors[9], as a measurement technique for the mass customisation of footwear, as a diagnostic tool for the development of new orthopaedic, orthotic and prosthetic devices, or the measurement of the pressure distribution beneath tyres [13]. There are also numerous other applications in science and engineering including the charactersation of the mechanical proper-

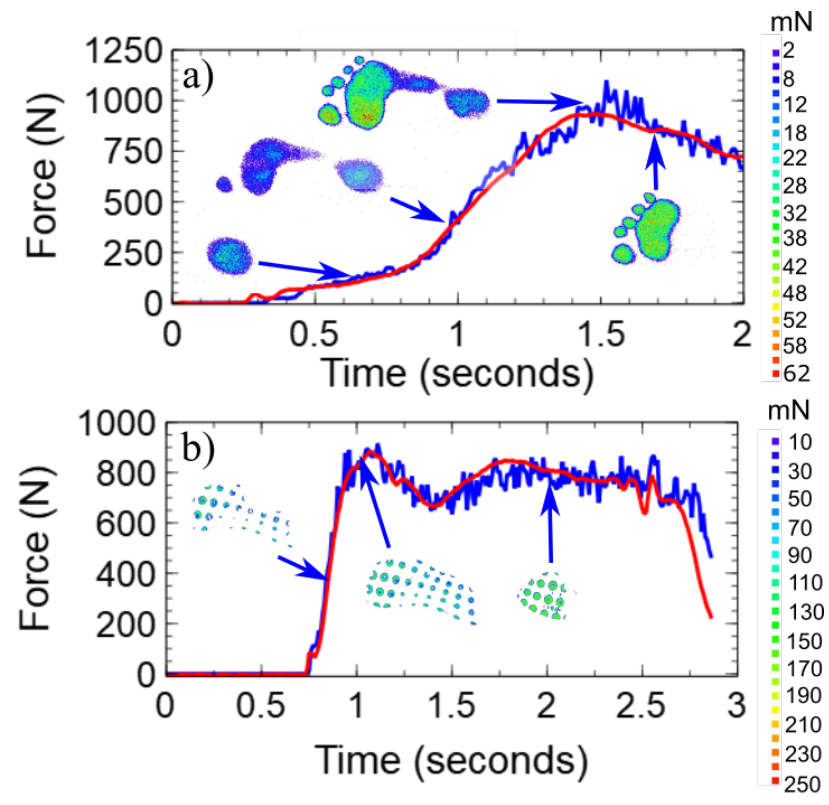

FIG. 10: FTIR imaging of footstrike events. Panel a) shows the variation in force exerted on the larger waveguide (see text) when one of the authors stepped up onto the FTIR imaging platform barefoot. Panel b) shows the variation in force when the same author repeated a similar experiment wearing a sports shoe. In both cases the blue lines represent the total force derived from the FTIR light scattering measurements and the red lines are the measurements obtained using the load cells. The colormap images give details of the local load distribution beneath the foot and the shoe respectively at different times. The colormap legends (right) show the equivalence between the colours and the local force detected in each pixel. All data was acquired at 100 frames/samples per second.

ties of materials (see figures 2 and 9), measurements of cell adhesion [27] as well as insect [8], animal [7] and human gait studies $[11,12]$. In each case, the optics and imaging element can be tailored to the application with relative ease.

One potential limitation of the FTIR imaging technique lies in the fact that intensity/force response needs to be calibrated and the $\beta$ value determined for each new material. If a contacting object is multi-coloured, or if it is made of a composite of materials with different Young's moduli then extracting meaningful force/pressure measurements could be difficult. The effects of having multicoloured objects are probably less of a concern if a prototype or mock-up of the contacting object can be made out of material having just a single colour. In the case of black materials (which scatter very little visible light) it is possible to excite the waveguide with infrared (IR) LEDs and to use an appropriate IR sensitive camera to image the contact regions. Having materials with dif- 
ferent Youngs moduli contacting the waveguide simultaneously would be more difficult to deal with and would require the development of more sophisticated measurement techniques and software analysis tools.

Application of the model presented above may also lead to a potential limitations if, for example, the contacting material has a higher refractive index than the waveguide. In this case, a modified version of the theory with an exponentially decaying intensity caused by scattering/attenuation of light transmitted into the contacting object would need to be used (as discussed previously). In addition, the theory described above assumes that the contacting object is more compliant than the waveguide surface. While this applies for all the systems studied here, the assumptions made in deriving the equations will start to break down if the contacting material has a Young's modulus that is comparable to, or greater than the waveguide material. Moreover, if the contacting material is made of a composite containing structures with different mechanical and optical responses this could make analysis of the data difficult. Under these circumstances, a simple average of these physical properties could be used to model the data (or be extracted from it depending upon the details of the experiment being performed). However, spatial variations in mechanical and/or optical properties within the contacting material could give rise to erroneous results if not properly accounted for. A priori information about the contacting material such as its mechanical properties and optical properties are necessary to utilize the model to obtain detailed quantitative comparisons with experimental results. However, these properties can be encompassed by a simple determination of the calibration factor, $\beta$, if the device is to be used simply as a pressure/force measurement tool (see above discussion). Even though this parameter needs to be determined for every material, it is relatively easy to extract from a single, simple loading experiment when using contacting materials with uniform mechanical and optical properties.

\section{CONCLUSIONS}

Frustrated total internal reflection imaging was used to measure the load/pressure distribution beneath soft objects when they contact a waveguide. The intensity response was shown to have a similar shape for contacting objects with different Young's moduli, surface roughness values and colours. In each case the intensity/force response curves were found to have a similar shape but to have a slope that was determined by a combination of these different factors. A theory was developed to explain the dependence of the light scattering response on the applied force and the physical (optical and mechanical) properties of the contacting materials. The experiments and theory developed in this work show that the scattered light intensity/force response can be calibrated so that each pixel on a camera can effectively be used as force sensor. The work here demonstrates that the measured force/pressure response is capable of reproducing the pressure distribution that is exerted beneath non-uniform contacting objects such as hemispherical indenters. The work also demonstrates that it is possible to map the load distributions beneath objects with more complicated geometries such as human feet and shoe outsoles and that the optically derived forces are in good agreement with forces measured by load cells. 


\section{ACKNOWLEDGMENTS}

We wish to acknowledge Adidas America Inc. for providing financial support for this project. We would also like to thank Dr. Long Jiang for performing the optical profilometry measurements on the dye doped elastomer pucks.

\section{Appendix A: Simplified Scattering model}

A simplified version of the scattering theory presented here can be made by approximating the strain field in the region of contact between an asperity and the waveguide surface. The functional form of $\epsilon_{z}$ can be made more simple by assuming that the strain is related to the local displacements at the surface of the asperity, $u_{z}$ such that

$$
\epsilon_{z} \sim \frac{u_{z}}{R}=\frac{3 F\left(1-\nu^{2}\right)}{8 E R a^{3}}\left(2 a^{2}-r^{2}\right)
$$

where the displacements at the interface are determined using Hertzian contact theory [15] and all other parameters are as defined in the main text.

In this case, the total scattering is given by combining equations 2, 3, 4 and $\mathrm{A} 1$ such that integration gives an expression for the scattering intensity from a single asperity as

$$
I_{s}=\frac{16 \pi^{4} I_{o} \alpha^{2}\left(1+\cos ^{2} \theta_{s}\right) \phi_{o}}{\lambda^{4} D^{2}} \int_{0}^{\infty} \exp \left(\frac{-4 \pi n_{o} \cos \theta_{r} z}{\lambda}\right) d z \int_{0}^{a} \frac{r}{1-(1-2 \nu) \frac{3 F\left(1-\nu^{2}\right)}{8 E R a^{3}}\left(2 a^{2}-r^{2}\right)} d r
$$

These integrals have an analytical solution which is given by

$$
I_{s}=\frac{4 \pi^{4} I_{o} \alpha^{2}\left(1+\cos ^{2} \theta_{s}\right) \phi_{o}}{\lambda^{3} D^{2}(1-2 \nu) n_{o} \cos \theta_{r}} R^{2} \ln \left(\frac{1-\frac{(1-2 \nu)}{2}\left(\frac{3 F\left(1-\nu^{2}\right)}{4 E R^{2}}\right)^{\frac{2}{3}}}{1-(1-2 \nu)\left(\frac{3 F\left(1-\nu^{2}\right)}{4 E R^{2}}\right)^{\frac{2}{3}}}\right)
$$

where again the substitution

$$
I_{p p}=I_{s} \frac{A_{p}}{4 R^{2}}
$$

$$
a=\left(\frac{3 F R\left(1-\nu^{2}\right)}{4 E}\right)^{\frac{1}{3}}
$$

has been made. The force and intensity per pixel can be approximated using the expressions

$$
F_{p p}=F \frac{A_{p}}{4 R^{2}}
$$

such that

$$
I_{p p}=\frac{\pi^{4} A_{p} I_{o} \alpha^{2}\left(1+\cos ^{2} \theta_{s}\right) \phi_{o}}{\lambda^{3} D^{2}(1-2 \nu) n_{o} \cos \theta_{r}} \ln \left(\frac{1-\frac{(1-2 \nu)}{2}\left(\frac{3 F_{p p}\left(1-\nu^{2}\right)}{E A_{p}}\right)^{\frac{2}{3}}}{1-(1-2 \nu)\left(\frac{3 F_{p p}\left(1-\nu^{2}\right)}{E A_{p}}\right)^{\frac{2}{3}}}\right)
$$

In the limit when $F_{p p}$ is small, i.e. when $F_{p p}<<\frac{E A_{p p}}{3\left(1-\nu^{2}\right)}$, equation A4 can be expanded to give a sim- 
ple power law dependence of the scattering intensity per pixel on the force exerted per pixel.

$$
I_{p p}=\frac{\pi^{4} A_{p} I_{o} \alpha^{2}\left(1+\cos ^{2} \theta_{s}\right) \phi_{o}}{2 \lambda^{3} D^{2} n_{o} \cos \theta_{r}}\left(\frac{3 F_{p p}\left(1-\nu^{2}\right)}{E A_{p}}\right)^{\frac{2}{3}}
$$

This equation predicts that the scattering intensity per pixel should be independent of the radius of the surface asperities and be sensitive to the Young's modulus of the surface asperities. This appears to be consistent with the observations shown in figures 3 and 4 .
[1] J. A. Needham and J. S. Sharp, "Watch your step! a frustrated total internal reflection approach to forensic footwear imaging," Scientific Reports 6, 21290 (2016).

[2] E. Hecht, Optics, Second Edition (Addison-Wesley, 1987) p. 104.

[3] S. Anantha Ramakrishna and A. D. Armour, "Propagating and evanescent waves in absorbing media," American Journal of Physics 71, 562 (2003).

[4] R. Sigel, "Light scattering near and from interfaces using evanescent wave and ellipsometric light scattering," Curr. Op. Coll. Int. Sci. 14, 426 (2009).

[5] S. Ramachandran, D. A. Cohen, A. P. Quist, and R. Lal, "High performance, led powered, waveguide based total internal reflection microscopy," Scientific Reports 3, 2133 (2013).

[6] A. I. Bennett, K. L. Harris, K. D. Schulze, J. Manuel Urueña, A J. McGhee, A. A. Pitenis, M. H. Müser, T. E. Angelini, and W. G. Sawyer, "Contact measurements of randomly rough surfaces," Tribol. Lett. 65, 134 (2017).

[7] D. H. Vrinten and F. F. T. Hamers, "Catwalk automated quantitative gait analysis as a novel method to assess mechanical allodynia in the rat; a comparison with von frey testing." Pain 102, 203 (2003).

[8] J. Kain, C. Stokes, Q. Gaudry, X. Song, J. Foley, R. Wilson, and B. de Bivort, "Leg-tracking and automated behavioural classification in drosophila," Nature Comms 4, 1910 (2013).

[9] T. Augsten, K. Kaefer, R. Meusel, C. Fetzer, D. Kanitz, T. Stoff, T. Becker, C. Holz, and P. Baudisch, "Multitoe: high-precision interaction with back-projected floors based on high-resolution multi-touch input," Proc.23rd ann. ACM symposium on User interface software and technology (UIST 10) , 209 (2010).

[10] N. D. Smith and J. S. Sharp, "Accessible biometrics: A frustrated total internal reflection approach to imaging fingerprints," Science and Justice 57, 193 (2017).

[11] R. P. Betts and T. A. Duckworth, "A device for measuring plantar pressures under the sole of the foot," Engineering in Medicine 7(4), 223 (1978).

[12] R. P. Betts, T. A. Duckworth, I. G. Austin, S. P. Crocker, and S. Moore, "Critical reflection at a plastic/glass interface and its application to foot pressure measurements," J. Med.Eng. Tech. 4(3), 136 (1980).

[13] J. J. C Aguilar, J. A. C Carrillo, A. J. G Fernandez, and S. P. Pogo, "Optimization of an optical test bench for tire properties measurements and tread defects charac- terisation," Sensors 17, 707 (2017).

[14] E. V. Eason, E. W. Hawkes, M. Windheim, D. L. Christensen, T. Libby, and M. R. Cutkosky, "Stress distribution and contact area measurements of a gecko toe using a high-resolution tactile sensor," Bioinspir. Biomim. 10, 016013 (2015).

[15] K. L. Johnson, Contact Mechanics (Cambridge University Press, 1985) p. 90.

[16] B. N. J. Persson, "Contact mechanics for randomly rough surfaces," Surface Science Reports 61, 201 (2006).

[17] M. Ciavarella, J. A. Greenwood, and M. Paggi, "Inclusion of interaction in the greenwood and williamson contact theory," Wear 265, 729 (2008).

[18] B. N. J. Persson, "Elastoplastic contact between randomly rough surfaces," Phys. Rev. Lett. 87(11), 116101 (2001).

[19] H. Driscoll, H. Koerger, T. Senior, and S. Haake, "The use of photoelasticity to identify surface shear stresses during running," Procedia Eng. 2, 3047 (2010).

[20] A. H. A Razak, A. Zayegh, R. K. Begg, and Y. Wahab, "Foot plantar pressure measurement system: A review," Sensors 12, 9884 (2012).

[21] I. D. Johnston, D. K. McCluskey, C. K. L. Tan, and M. C. Tracey, "Mechanical characterization of bulk sylgard 184 for microfluidics and microengineering," J. Micromech. Microeng. 24, 035017 (2014).

[22] M. Ochsner, M. R. Dusseiller, H. M. Grandin, S. LunaMorris, M. Textor, V. Vogel, and M. L. Smith, "Microwell arrays for $3 \mathrm{~d}$ shape control and high resolution analysis of single cells," Lab Chip 7, 1074 (2007).

[23] C. S. Johnson and D. A. Gabriel, Laser Light Scattering (Dover, 1981) p. 3.

[24] D. A. Hills, D. Nowell, and A. Sackfield, Mechanics of Elastic Contacts (Butterworth-Heinneman, 1993) p. 203.

[25] J. Brandrup, E. H. Immergut, and E. A. Grulke Eds., Polymer Handbook, 4th Edition (Wiley, 1999) pp. V1-8.

[26] P. S. D. Patel, D. E. T. Shepherd, and D. W. L. Hukins, "Compressive properties of commercially available polyurethane foams as mechanical models for osteoporotic human cancellous bone," BMC Musculoskeletal Disorders 9, 137 (2008).

[27] G. D. Byrne, M. C. Pitter, J. Zhang, F. H. Falcone, S. Stolnik, and M. G. Somekh, "Total internal reflection microscopy for live imaging of cellular uptake of submicron nonfluorescent particles," J. Microscopy 231(1), 168 (2008). 


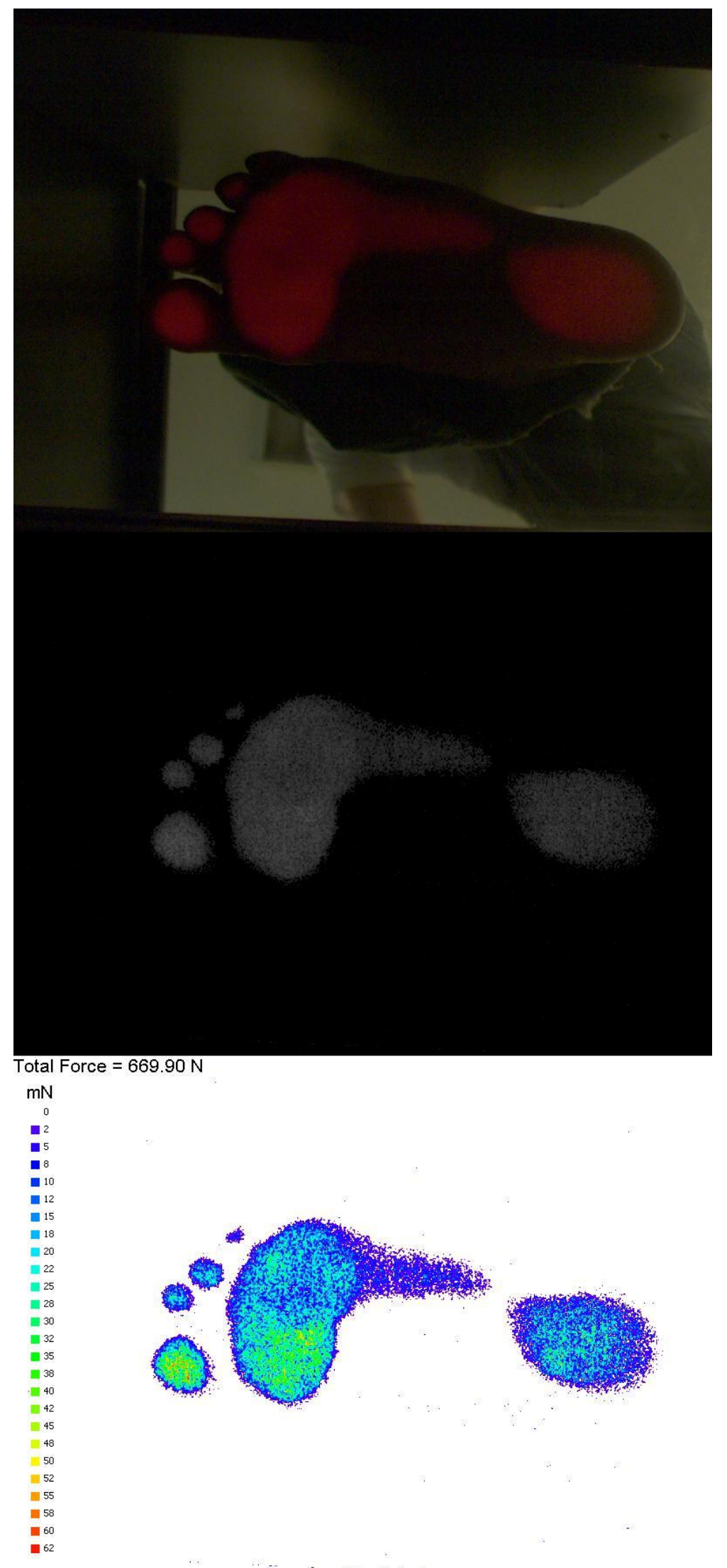

Video 1. Composite movie showing raw FTIR image data (top panel), background subtracted data (middle panel) and calculated force data (bottom panel) during a footstrike event involving a bare foot. 


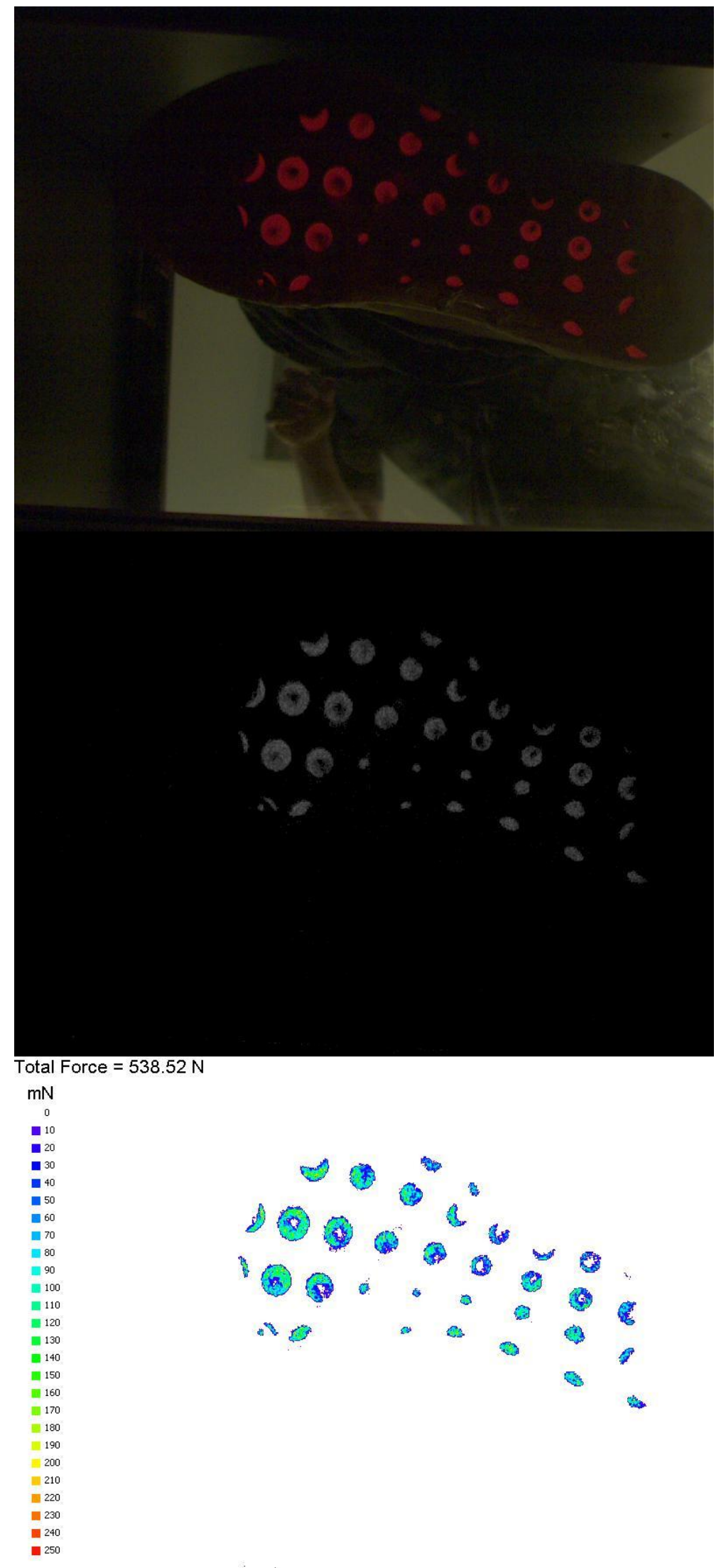

Video 2. Composite movie showing raw FTIR image data (top panel), background subtracted data (middle panel) and calculated force data (bottom panel) during a footstrike event involving a sports shoe. 